\title{
ERGE persistente o recidivante luego de cirugía antirreflujo, síntomas que Ilevan a la reintervención en un hospital privado de la Ciudad de México
}

\author{
Persistent or recurrent GERD after antireflux \\ surgery, symptoms leading to reoperation \\ in a private hospital in Mexico City
}

\author{
Rodolfo Iván Lara Ruiz, * Itzel Alejandra García Anzures, ${ }^{\ddagger}$ Jorge Chirino Romo ${ }^{\S}$ \\ Citar como: Lara RRI, García AIA, Chirino RJ. ERGE persistente o recidivante luego de cirugía \\ antirreflujo, síntomas que llevan a la reintervención en un hospital privado de la Ciudad de México.
}

Acta Med GA. 2022; 20 (1): 17-23. https://dx.doi.org/10.35366/103551

\section{Resumen}

La persistencia o recidiva de síntomas posteriores a cirugía antirreflujo se asocian al estudio y abordaje inicial deficientes, fallas en la técnica quirúrgica y factores de riesgo propios del paciente. Con el objetivo de describir los síntomas que llevan a una nueva intervención, tiempo desde el primer procedimiento, técnica empleada y características de los casos, se realizó una revisión sistemática retrospectiva de 100 expedientes clínicos sometidos a reintervención de cirugía antirreflujo durante los últimos cuatro años en el Hospital Angeles Pedregal en la Ciudad de México. Se encontraron casos con hasta tres reintervenciones, los resultados mostraron que pirosis $(65 \%)$, regurgitación (58\%) y epigastralgia $(28 \%)$ fueron los síntomas más frecuentes. El factor de riesgo aislado más importante fue la obesidad (23\%). Más de $50 \%$ se reintervinieron dentro de los primeros 10 años, siendo el deslizamiento de la funduplicatura el mecanismo de falla más común (71\%). Si bien los resultados coinciden en gran medida con la literatura, la comparación sistemática de los casos con dos o más cirugías como en nuestra muestra ha sido reportada en pocos estudios en la era laparoscópica, por lo que el presente trabajo contribuye al análisis de casos con múltiples tratamientos quirúrgicos antirreflujo.

Palabras clave: Funduplicatura, recurrencia, reoperación, falla terapéutica, complicaciones postquirúrgicas, factores de riesgo.

\section{Abstract}

The persistence or recurrence of symptoms after antireflux surgery is associated with poor study and initial approach, failures in surgical technique and risk factors specific to the patient. In order to describe the symptoms that lead to a new intervention, time since the first procedure, the technique used and the characteristics of the patients, a retrospective systematic review of 100 clinical records of cases undergoing reintervention of antirreflux surgery during the last four years was carried out in Angeles Pedregal Hospital in Mexico City. Cases with up to three previous surgeries were found, the results showed that heartburn $(65 \%)$, regurgitation $(58 \%)$ and epigastric pain $(28 \%)$ were the most frequent symptoms. The most important isolated risk factor was obesity $(23 \%)$. More than $50 \%$ underwent reoperation within the first 10 years, with slippage of the fundoplication being the most common failure mechanism (71\%). Although the results largely coincide with the literature, the systematic comparison of cases with two or more surgeries as in our sample has been reported in few studies in the laparoscopic era, so the present work contributes to the analysis of cases with multiple anti-reflux surgical treatments.

Keywords: Fundoplication, recurrence, reoperation, treatment failure, postoperative complications, risk factors.

\footnotetext{
* Residente de cuarto año de Cirugía General. Facultad Mexicana de Medicina de la Universidad La Salle.

₹ Residente de segundo año de Cirugía General. Facultad Mexicana de Medicina de la Universidad La Salle.

§ Médico Especialista en Cirugía General y Nutriología Clínica.
}

Hospital Angeles Pedregal. México.
Correspondencia:

Rodolfo Iván Lara Ruiz

Correo electrónico: ivanruiz.cirugia@gmail.com

Aceptado: 24-04-2021.

www.medigraphic.com/actamedica

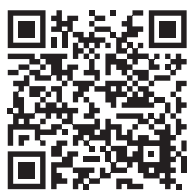




\section{INTRODUCCIÓN}

En la actualidad, la cirugía tiene un lugar establecido en el manejo de la enfermedad por reflujo gastroesofágico (ERGE). La popularización de la resolución quirúrgica abierta, y después por mínima invasión, ha permitido la globalización de la funduplicatura laparoscópica, estableciéndose como el estándar de oro en el manejo quirúrgico de ERGE con excelentes resultados y remisión sintomática a largo plazo en $90-95 \%$ de los casos. ${ }^{1-5}$

El objetivo del tratamiento quirúrgico es restablecer la barrera antirreflujo, proteger la mucosa esofágica evitando el reflujo ácido y la prevención de complicaciones. ${ }^{6}$ La funduplicatura se realiza como componente de la barrera antirreflujo; sin embargo, su papel en la prevención de la recurrencia aún no está claro. El cirujano sólo cuenta con el control parcial sobre este hecho mediante una adecuada técnica quirúrgica. Los elementos esenciales de una funduplicatura exitosa incluyen la reparación de la hernia hiatal y la plicatura infradiafragmática libre de tensión alrededor del esófago distal con una longitud de al menos 2-3 cm de esófago intraabdominal.7,8

La persistencia de síntomas gastroesofágicos ha sido considerada sinónimo de falla quirúrgica, además de ser una indicación clara para reintervenir. ${ }^{9}$ Las razones de falla están bien documentadas, por lo general se deben al pobre abordaje del paciente, selección incorrecta del tipo de procedimiento, errores técnicos o incluso deterioro de la motilidad esofagogástrica. ${ }^{10}$ Los factores anatómicos que ameritan revisión quirúrgica son el deslizamiento de la funduplicatura, plicatura muy ajustada, dehiscencia de la misma o hernia hiatal recurrente, siendo la migración intratorácica el fallo más frecuente. ${ }^{11,12}$ Entender qué es lo que lleva a la falla es crítico para el éxito de una posible reintervención.

El índice de recurrencia radiográfica se reporta en 12$16 \%$ con recurrencia sintomática que amerita una o más reintervenciones en $3-11 \%$ de los casos. ${ }^{13}$

Las reintervenciones sobre el hiato esofágico son más complejas y no siempre se acompañan de respuesta clínica satisfactoria, tienen mayor riesgo de lesiones iatrogénicas y al mismo tiempo incrementan la posible necesidad de una nueva intervención en 3-16\% de los pacientes, ${ }^{11}$ por lo que es necesario establecer expectativas realistas en cuanto a los resultados funcionales esperados con los pacientes con una o más cirugías previas antirreflujo.

El objetivo del estudio fue describir los principales síntomas compatibles con persistencia o recidiva de ERGE y la posible relación con los factores perioperatorios de cada paciente, así como analizar el tiempo desde la cirugía previa, el tipo de reparación, los hallazgos y frecuencia de complicaciones intraoperatorias en casos reintervenidos en el Hospital Angeles Pedregal.

\section{MATERIAL Y MÉTODOS}

Se realizó una revisión retrospectiva de 100 expedientes con diagnóstico al ingreso de ERGE persistente o recidivante con funduplicatura previa que ameritaron reintervención por diferentes equipos quirúrgicos en el periodo comprendido de marzo 2016 a marzo 2020 en el Hospital Angeles Pedregal.

Los datos recabados incluyeron los síntomas asociados con recidiva de la enfermedad, edad al momento de la primera cirugía, IMC, comorbilidades crónico-degenerativas, número de procedimientos antirreflujo previos, intervalo entre procedimientos, técnicas quirúrgicas, así como los hallazgos y complicaciones intraoperatorias reportadas. Los pacientes provenían de diversas unidades hospitalarias y fueron abordados por diferentes equipos quirúrgicos dentro del Hospital Angeles Pedregal.

\section{RESULTADOS}

De marzo de 2016 a marzo de 2020 se recabaron 100 reintervenciones por síntomas persistentes o recidivantes de reflujo gastroesofágico con cirugía antirreflujo previa realizada por mínima invasión en diversos centros hospitalarios. Se trató de la primera reintervención en el Hospital Angeles Pedregal en 87 casos (87\%), segunda en 11 (11\%) y tercera en dos $(2 \%)$.

La muestra estuvo conformada por 47 hombres (47\%) y 53 mujeres (53\%) con edad promedio de 50.3 años (15-91). La edad promedio fue mayor en los casos con dos reintervenciones, 60.5 años (48-91). El peso promedio al momento de la última reintervención fue de $73.1 \mathrm{~kg}$ (39-112) con un IMC (índice de masa corporal) de 26.2 (12-45.4), siendo mayor en el grupo con dos reintervenciones (28.1). Las comorbilidades registradas incluyeron: obesidad IMC > 30 (23\%), hipertensión arterial (21\%), hipotiroidismo (13\%), diabetes mellitus tipo 2 (5\%) y Ehlers-Danlos (1\%) (Tabla 1).

Los síntomas referidos incluyeron: pirosis (65\%), regurgitación (58\%), epigastralgia (28\%), síntomas respiratorios (23\%), náusea o vómito (20\%), disfagia (13\%), dolor abdominal $(11 \%)$, hiporexia o anorexia $(7 \%)$ e intolerancia a la vía oral (3\%) (Tabla 2). La pirosis fue el síntoma predominante en los pacientes con una reintervención, mientras que la regurgitación lo fue en los casos con dos.

El intervalo de tiempo promedio desde la última cirugía antirreflujo fue de 6.2 años (1-23) para la primera reintervención, 8.1 años (2-30) para la segunda y cuatro años (2-6) para la tercera, $12 \%$ se sometió a reintervención dentro del primer año, 39\% a los cinco años y más de $50 \%$ a los 10 años de la última cirugía antirreflujo (Tabla 3).

No fue posible recabar los detalles del procedimiento realizado en la primera cirugía antirreflujo en todos los ca- 
Tabla 1: Características de los pacientes sometidos a redo comparados por el número de reintervención.

\begin{tabular}{|c|c|c|c|c|}
\hline & \multirow[b]{2}{*}{ Total $(N=100)$} & \multicolumn{3}{|c|}{ Número de reintervención } \\
\hline & & Primera $(\mathrm{N}=87)$ & Segunda $(N=11)$ & Tercera $(\mathrm{N}=2)$ \\
\hline Edad (años)* & $50.3(15-91)$ & $49.1(15-81)$ & $60.5(48-91)$ & $43(20-66)$ \\
\hline \multicolumn{5}{|l|}{ Género, n (\%) } \\
\hline Masculino & $47(53)$ & $40(40)$ & $5(5)$ & $2(2)$ \\
\hline Femenino & $53(47)$ & $47(47)$ & $6(6)$ & $0(0)$ \\
\hline $\operatorname{IMC}\left(\mathrm{kg} / \mathrm{m}^{2}\right)^{*}$ & $26.2(12-45.4)$ & $26.1(14.5-36.1)$ & $28.1(14.2-38.2)$ & $22.3(19.7-24.9)$ \\
\hline
\end{tabular}

Tabla 2: Síntomas referidos compatibles con persistencia o recidiva de ERGE.

\begin{tabular}{|c|c|c|c|c|}
\hline \multirow[b]{2}{*}{ Síntomas } & \multirow[b]{2}{*}{ Total $(N=100)$} & \multicolumn{3}{|c|}{ Número de reintervención, n (\%) } \\
\hline & & Primera $(\mathrm{N}=87$ ) & Segunda $(N=11)$ & Tercera $(\mathrm{N}=2)$ \\
\hline Pirosis & $65(65)$ & $58(66.6)$ & $5(45.4)$ & $2(100)$ \\
\hline Regurgitación & $58(58)$ & $48(55.1)$ & $8(72.7)$ & $2(100)$ \\
\hline Epigastralgia & $28(28)$ & $26(29.8)$ & $1(9.0)$ & $1(50)$ \\
\hline Síntomas respiratorios* & $23(23)$ & 20 (22.9) & $3(27.2)$ & - \\
\hline Náusea/vómito & $20(20)$ & $16(18.3)$ & $3(27.2)$ & $1(50)$ \\
\hline Disfagia & $13(13)$ & $11(12.6)$ & $2(18.1)$ & - \\
\hline Distensión/dolor abdominal & $11(11)$ & $11(12.6)$ & - & - \\
\hline Anorexia/hiporexia & $7(7)$ & $5(5.7)$ & $1(9.0)$ & $1(50)$ \\
\hline Intolerancia a la vía oral & $3(3)$ & $3(3.4)$ & - & - \\
\hline
\end{tabular}

\section{Tabla 3: Intervalo entre cirugías.}

\begin{tabular}{ccccc} 
& & \multicolumn{2}{c}{ Número de reintervención, $\mathrm{n}(\%)$} \\
\cline { 3 - 4 } Intervalo en años & Total $(\mathrm{N}=100)$ & Primera $(\mathrm{N}=87)$ & Segunda $(\mathrm{N}=11)$ & Tercera $(\mathrm{N}=2)$ \\
\hline$<1$ & $12(12)$ & $12(13.7)$ & $5(45.4)$ & - \\
$2-5$ & $27(27)$ & $21(24.1)$ & $1(9.0)$ & $1(50)$ \\
$6-10$ & $27(27)$ & $25(28.7)$ & $1(9.0)$ & - \\
$11-15$ & $9(9)$ & $8(9.1)$ & $1(9.0)$ & - \\
$>16$ & $3(3)$ & $2(2.2)$ & $3(27.2)$ & - \\
\hline
\end{tabular}

* Intervalo no especificado con respecto al último procedimiento antirreflujo. 
sos, ya que los pacientes provenían de diferentes hospitales. Mismo caso con los hallazgos intraoperatorios durante las reintervenciones realizadas en el Hospital Angeles Pedregal donde participaron diferentes equipos quirúrgicos.

Los estudios de gabinete incluyeron endoscopia (68\%), esofagograma (48\%), manometría (16\%) y pHmetría (4\%). Sólo un caso para la primera reintervención contó con los cuatro estudios (Tabla 4). El hallazgo por endoscopia más común fue el deslizamiento de la funduplicatura con recidiva de la hernia hiatal en 27 casos $(27 / 68,39.7 \%)$, por esofagograma el reflujo de material de contraste hasta el tercio medio con deslizamiento de unión gastroesofágica por arriba del diafragma en 15 casos (15/48, 31.2\%), por manometría el esfínter esofágico inferior incompetente en longitud y presión en ocho casos $(8 / 16,50 \%)$ y tres pHmetrías resultaron positivas $(3 / 4,75 \%)$.

Todas las reintervenciones se iniciaron y completaron laparoscópicamente, 93 se resolvieron mediante redo de la funduplicatura con técnicas Nissen o Toupet, para los siete casos restantes se realizaron otras técnicas: Hill, Dor, Gea, bypass gástrico en Y de Roux y Billroth II (Tabla 5). Los hallazgos transoperatorios reportados incluyeron hernia hiatal recidivante $(37 \%)$ y deslizamiento de la funduplicatura (10\%), para el resto de los casos (53\%) no se encontró el reporte detallado de los hallazgos transoperatorios.

La decisión del tipo de reparación se vio influenciada por los hallazgos intraoperatorios, el grado de disrupción de la anatomía y el campo visual secundario a la presencia de adherencias. El redo tipo Nissen fue el procedimiento más realizado en los grupos de la primera $(74.7 \%, 65 / 87)$ y segunda $(63.6 \%, 7 / 11)$ reintervenciones. Las técnicas aisladas fueron realizadas con base en la decisión particular del equipo quirúrgico.

El uso de malla se reportó en cinco procedimientos (5\%) reparados mediante redo Nissen. Del total de procedimientos, dos se realizaron asistidos por robot (DaVinci SiHD), el procedimiento tipo Dor y un redo Nissen por decisión del equipo quirúrgico.

El tiempo quirúrgico promedio fue de 162 minutos (60480). El menor tiempo (60 min) fue para un redo Nissen con plastia de hiato, mientras que el mayor (480 min) correspondió a una funduplicatura deslizada con estómago intratorácico que ameritó toracotomía para la liberación de adherencias y la reparación mediante redo Nissen con plastia de hiato (Tabla 5).

Las ocho complicaciones intraoperatorias reportadas se muestran en la Tabla 6. Se reportaron seis perforaciones gástricas durante el desmantelamiento de la funduplicatura, cuatro durante la primera reintervención. Los seis casos se repararon laparoscópicamente, tres mediante gastrectomía parcial y tres con cierre primario del defecto. Además, se reportaron dos estenosis luego de redo Nissen, un caso después de la primera reintervención, el otro luego de la tercera, ambos casos se sometieron a dilatación neumática. Ninguna de las ocho complicaciones ameritó conversión a laparotomía. Al tratarse de un estudio retrospectivo, el seguimiento de los pacientes no estuvo dentro de los objetivos.

Tabla 4: Estudios de gabinete.

\begin{tabular}{|c|c|c|c|c|}
\hline \multirow[b]{2}{*}{ Estudio de gabinete } & \multirow[b]{2}{*}{ Total $(N=100)$} & \multicolumn{3}{|c|}{ Número de reintervención, n (\%) } \\
\hline & & Primera $(\mathrm{N}=87)$ & Segunda $(N=11)$ & Tercera $(\mathrm{N}=2)$ \\
\hline Endoscopia & $17(17)$ & $16(18.3)$ & $1(9.0)$ & - \\
\hline Esofagograma & $13(13)$ & $11(12.6)$ & $1(9.0)$ & $1(50)$ \\
\hline Manometría esofágica & - & - & - & - \\
\hline pHmetría & - & - & - & - \\
\hline $\begin{array}{l}\text { Endoscopia + } \\
\text { esofagograma }\end{array}$ & $33(33)$ & $28(32.1)$ & $4(36.3)$ & $1(50)$ \\
\hline Endoscopia + manometría & $14(14)$ & $11(12.6)$ & $3(27.2)$ & - \\
\hline $\begin{array}{l}\text { Endoscopia + } \\
\text { esofagograma + pHmetría }\end{array}$ & $2(2)$ & $1(1.1)$ & $1(9.0)$ & - \\
\hline $\begin{array}{l}\text { Endoscopia + manometría + } \\
\text { pHmetría }\end{array}$ & $2(2)$ & $2(2.2)$ & - & - \\
\hline Ningún estudio & $19(19)$ & - & - & - \\
\hline
\end{tabular}


Tabla 5: Tipo de reparación y tiempo quirúrgico.

\begin{tabular}{|c|c|c|c|c|}
\hline \multirow[b]{2}{*}{ Procedimiento } & \multirow[b]{2}{*}{ Total $(N=100)$} & \multicolumn{3}{|c|}{ Número de reintervención, n (\%) } \\
\hline & & Primera $(\mathrm{N}=87)$ & Segunda $(N=11)$ & Tercera $(\mathrm{N}=2)$ \\
\hline Duración $(\min )^{*}$ & $162(60-480)$ & $154.4(60-480)$ & $203.6(120-450)$ & $285(170-400)$ \\
\hline Nissen & $73(73)$ & $65(74.7)$ & $7(63.6)$ & $1(50)$ \\
\hline Toupet & $20(20)$ & $17(19.5)$ & $3(27.2)$ & - \\
\hline Toupet $+Y$ de Roux & $1(1)$ & $1(1.1)$ & - & - \\
\hline Plastia de hiato & $1(1)$ & - & - & $1(50)$ \\
\hline Dor & $1(1)$ & $1(1.1)$ & - & - \\
\hline Hill & $1(1)$ & - & $1(9.0)$ & - \\
\hline Gea** & $1(1)$ & $1(1.1)$ & - & - \\
\hline Billroth II & $1(1)$ & $1(1.1)$ & - & - \\
\hline Y de Roux & $1(1)$ & $1(1.1)$ & - & - \\
\hline
\end{tabular}

Tabla 6: Complicaciones transoperatorias.

\begin{tabular}{|c|c|c|c|c|}
\hline \multirow[b]{2}{*}{ Complicación } & \multirow[b]{2}{*}{ Total $(N=100)$} & \multicolumn{3}{|c|}{ Número de reintervención, n (\%) } \\
\hline & & Primera $(\mathrm{N}=87)$ & Segunda $(N=11)$ & Tercera $(\mathrm{N}=2)$ \\
\hline Perforación gástrica & $6(6)$ & $4(4.5)$ & $1(9.0)$ & $1(50)$ \\
\hline Estenosis postfunduplicatura & $2(2)$ & $1(1.1)$ & $1(9.0)$ & - \\
\hline
\end{tabular}

\section{DISCUSIÓN}

La recurrencia sintomática luego de la cirugía antirreflujo es una complicación donde factores técnicos propios al procedimiento quirúrgico y asociados al paciente se relacionan con la falla del procedimiento.

En cuanto a la evolución natural de la enfermedad, la edad cada vez mayor juega un papel importante en la necesidad de una o más cirugías antirreflujo, como en este caso al comparar las edades promedio en años (60 contra 49), siendo mayor en el grupo con dos reintervenciones. El grupo de la tercera reintervención se conformó sólo por dos casos con edades muy distantes ( 20 y 66) que dificultaron una asociación más certera.

Dentro de los factores de riesgo perioperatorios, el único independiente asociado claramente a la falla de la cirugía es la obesidad, coincidiendo en nuestra muestra dentro del grupo con más de una reintervención (IMC 28.1) en comparación con el grupo de una (IMC 26.2) y tres cirugías
(IMC 22.3). Se mencionan otros posibles factores como la presencia de síntomas atípicos, falta de respuesta a medicamentos, tos crónica, náusea, pobre peristaltismo esofágico preoperatorio, hernia hiatal grande, género femenino e incluso edad mayor a 50 años, ${ }^{4,5,9}$ por lo que el abordaje inicial en estos casos cobra mayor importancia.

El tipo de síntomas reportados como compatibles con la persistencia o recidiva coinciden con los reportados en otros estudios, que además analizan el impacto en la calidad de vida y la mejoría de los mismos posterior a la reintervención, siendo los más comunes la pirosis, reflujo y epigastralgia.

El abordaje integral es crucial en la búsqueda de la causa y para la selección del tratamiento apropiado. Este proceso incluye un interrogatorio completo, indagar en detalles del procedimiento previo, así como el uso de estudios de gabinete como endoscopia alta, esofagograma, pHmetría o manometría esofágica en la mayoría de los casos. ${ }^{5}$ La indicación de reintervención se recomienda cuando se 
evidencia una anomalía anatómica o funcional corregible por cirugía que corresponde con los síntomas. ${ }^{8,12}$

Existen algoritmos de abordaje en aquéllos con una o más cirugías antirreflujo; ${ }^{14}$ sin embargo, los antecedentes, tiempo de evolución y factores de riesgo detectados hacen del estudio un proceso dinámico, de ahí la variación en cuanto al tipo de estudios de gabinete realizados en nuestra muestra, siendo la endoscopia y el esofagograma los más solicitados de manera inicial y en muchas ocasiones los únicos necesarios para tomar una decisión terapéutica.

La relación entre el tipo de procedimiento inicial y la frecuencia de recidiva sintomática podría arrojar resultados interesantes, aunque no fue posible realizarla en nuestra muestra dado que no contamos con los detalles técnicos del primer procedimiento en todos los casos, además de la participación de múltiples equipos quirúrgicos y la concentración de personas provenientes de diversos hospitales.

Está claro que el tiempo quirúrgico se verá afectado por el número de intervenciones previas dado el proceso adherencial resultante, el grado de disrupción de la anatomía y la experiencia del equipo quirúrgico.

El riesgo de recurrencia de hernia hiatal se ha reportado igual de alto en reparados con o sin malla, ${ }^{4}$ por lo que su uso podría ser una decisión subjetiva dentro de algunos equipos quirúrgicos, siendo empleada sólo en cinco casos dentro de nuestra muestra.

Dado que el grado de satisfacción posterior a cada reintervención es menor en comparación con la primera cirugía, reportándose en hasta $81 \%, 13,14$ no es raro encontrar casos con tres o más procedimientos que persisten sintomáticos, por lo que el tipo de reparación se convierte en el punto cardinal determinante de la posibilidad de recidiva. Al día de hoy, no existe un número particular de intervenciones de la unión esófago-gástrica a partir del cual una cirugía más esté contraindicada. ${ }^{14}$ Técnicas derivativas como la Y de Roux o Billroth II cobran especial importancia en aquéllos con múltiples factores de riesgo como obesidad mórbida, dismotilidad esofágica, esófago corto, disfagia severa o más de una reintervención. 4,13

La comparación sistemática entre la primera cirugía antirreflujo y cada procedimiento subsecuente ha sido reportada en pocos estudios en la era laparoscópica, ${ }^{4}$ por lo que consideramos el presente trabajo como un contribuyente más al estudio de los resultados de múltiples intervenciones quirúrgicas en el tratamiento de ERGE recidivante o persistente.

Es importante recordar que cualquier reintervención se asocia con mayor morbilidad en comparación con la primera cirugía antirreflujo, con complicaciones intraoperatorias reportadas hasta en $10-20 \%$, siendo la perforación gástrica o esofágica durante la lisis de adherencias las más frecuentes, así como complicaciones postoperatorias hasta en 15\% que incluyen lesión pleural, fuga gastrointestinal, infección de herida, entre otras. ${ }^{5}$

\section{CONCLUSIÓN}

La persistencia, recurrencia o nueva aparición de síntomas luego de un procedimiento antirreflujo amerita en todos una evaluación exhaustiva de la anatomía y funcionalidad esófago-gástrica previo a la decisión de manejo quirúrgico.

La primera cirugía antirreflujo es la mejor oportunidad para conseguir el alivio prolongado de los síntomas, por lo que debe realizarse con extrema atención a cada detalle idealmente en centros especializados a cargo de equipos experimentados con la finalidad de optimizar los resultados; sin embargo, no es un procedimiento infalible, la falla anatómica se ha reportado como la principal causa de recurrencia asociada a factores propios y ajenos a cada individuo.

La indicación para una nueva cirugía, el momento adecuado y la selección del tipo de reparación, sin dejar de lado la calidad de la técnica quirúrgica, determinarán la incidencia de éxito o falla subsecuente.

Limitaciones: reconocemos en primer lugar el carácter retrospectivo del trabajo, el número de pacientes, la necesidad de estandarización de los registros en el expediente clínico, así como la falta de un abordaje médico y quirúrgico homogéneo dada la intervención de múltiples equipos dentro y fuera del hospital. Por lo que la necesidad de un seguimiento más completo y a largo plazo es recomendable como complemento al presente trabajo.

\section{REFERENCIAS}

1. GPC. Diagnóstico y tratamiento de la ERGE en el adulto. Actualización 2018.

2. Prieto-Díaz-Chávez E, Medina-Chávez JL, Brizuela-Araujo CA, González-Jiménez MA, Mellín-Landa TE, Gómez-García TS et al. Calidad de vida y grado de satisfacción de pacientes postoperados de funduplicatura Nissen laparoscópica. Rev Gastroenterol Mex. 2014; 79 (2): 73-78.

3. Granderath FA, Kamolz T, Schweiger UM, Pointner R. Failed antireflux surgery: quality of life and surgical outcome after laparoscopic refundoplication. Int J Colorectal Dis. 2003; 18 (3): 248-253.

4. Singhal S, Kirkpatrick DR, Masuda T, Gerhardt J, Mittal SK. Primary and redo antireflux surgery: outcomes and lessons learned. J Gastrointest Surg. 2018; 22 (2): 177-186.

Munie S, Nasser H, Gould JC. Salvage options for fundoplication failure. Curr Gastroenterol Rep. 2019; 21 (9): 41.

6. Kamolz T, Bammer T, Wykypiel H Jr, Pasiut M, Pointner R. Quality of life and surgical outcome after laparoscopic Nissen and Toupet fundoplication: one-year follow-up. Endoscopy. 2000; 32 (5): 363-368.

7. Ellis R, Garwood G, Khanna A, Harmouch M, Miller CC, Banki F. Patient-related risk factors associated with symptomatic recurrence requiring reoperation in laparoscopic hiatal hernia repair. Surg Open Sci. 2019; 1 (2): 105-110.

8. O Ohnmacht GA, Deschamps C, Cassivi SD, Nichols FC 3rd, Allen MS, Schleck CD et al. Failed antireflux surgery: results after 
reoperation. Ann Thorac Surg. 2006; 81 (6): 2050-2053; discussion 2053-2054.

9. Grover BT, Kothari SN. Preoperative antireflux surgery. Surg Clin North Am. 2015; 95 (3): 629-640.

10. Hunter JG, Smith CD, Branum GD, Waring JP, Trus TL, Cornwall M et al. Laparoscopic fundoplication failures: patterns of failure and response to fundoplication revision. Ann Surg. 1999; 230 (4): 595604.

11. Ortíz I, Targarona EM, Pallares LL, Marinello F, Balague C, Trias M. Calidad de vida y resultados a largo plazo de las reintervenciones efectuadas por laparoscopia tras cirugía de hiato esofágico. Rev Cir Esp. 2009; 86 (2): 72-78.

12. Awais O, Luketich JD, Schuchert MJ, Morse CR, Wilson J, Gooding WE et al. Reoperative antireflux surgery for failed fundoplication: an analysis of outcomes in 275 patients. Ann Thorac Surg. 2011; 92 (3): 1083-1089; discussion 1089-1090.
13. Yamamoto SR, Hoshino M, Nandipati KC, Lee TH, Mittal SK. Longterm outcomes of reintervention for failed fundoplication: redo fundoplication versus Roux-en-Y reconstruction. Surg Endosc. 2014; 28 (1): 42-48

14. Antiporda M, Jackson C, Smith CD, Thomas M, Elli EF, Bowers SP. Strategies for surgical remediation of the multi-fundoplication failure patient. Surg Endosc. 2019; 33 (5): 1474-1481.

15. Bahena-Aponte JA, Moreno Portillo M, Ortega Larrocea X, Pérez Cruz RO, Guerrero Becerril LE, Flores Gama F. Estado actual de la enfermedad por reflujo gastroesofágico y su tratamiento quirúrgico. Rev Hosp Gral Dr. M Gea González. 2007; 8 (1): 25-33.

Conflicto de intereses: No existe conflicto de intereses por parte de ninguno de los autores implicados en la elaboración del presente artículo. 